
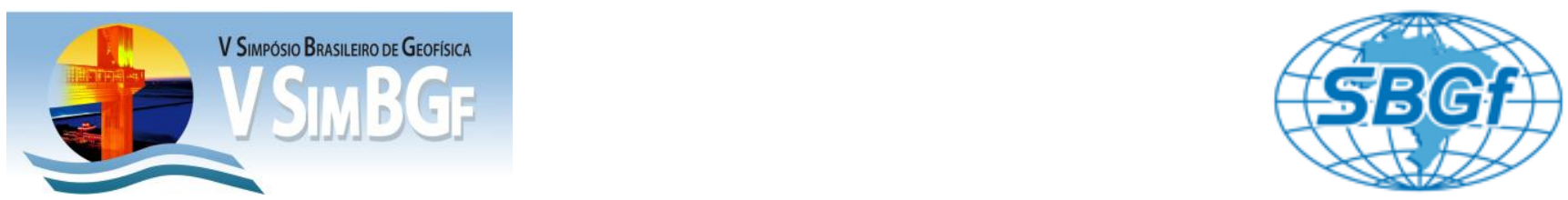

\title{
Datação arqueomagnética no Engenho Central de Piracicaba, São Paulo
}

Guilherme S. Begnini* - guilherme.begnini@gmail.com, Gelvam A. Hartmann, Ricardo I. F. Trindade

Universidade de São Paulo - Instituto de Astronomia, Geofísica e Ciências Atmosféricas - Departamento de Geofísica -

Laboratório de Paleomagnetismo

Copyright 2012, SBGf - Sociedade Brasileira de Geofísica

Este texto foi preparado para a apresentação no V Simpósio Brasileiro de Geofísica, Salvador, 27 a 29 de novembro de 2012. Seu conteúdo foi revisado pelo Comitê Técnico do V SimBGf, mas não necessariamente representa a opinião da SBGf ou de seus associados. É proibida a reprodução total ou parcial deste material para propósitos comerciais sem prévia autorização da SBGf.

\section{Resumo}

O campo magnético da Terra (CMT) pode ser estudado a partir do registro magnético em materiais arqueológicos que portam uma magnetização remanente estável. A utilização desses registros permite entender a evolução do CMT no passado e aplicar em datação arqueológica. A "datação arqueomagnética" consiste na comparação de um dado magnético de um material de idade desconhecida com a "curva de referência" para uma região do planeta, determinada pelos dados arqueomagnéticos e/ou modelos do CMT. Neste trabalho foi realizada datação arqueomagnética a partir da inclinação magnética remanente em tijolos do Engenho Central de Piracicaba, sendo possível datar edificações de idade desconhecida.

\section{Introdução}

As evidências sobre a origem e a evolução do campo magnético da Terra (CMT) podem somente ser acessadas através de medidas magnéticas diretas e indiretas, feitas na superfície da Terra ou acima dela. As medidas indiretas do CMT são determinadas a partir da magnetização remanente registrada em materiais arqueológicos e geológicos. Para obtenção de dados direcionais indiretos (declinação e inclinação) do CMT é necessário conhecer a posição em que o objeto adquiriu a magnetização e a amostragem deve ser feita in situ em amostras orientadas (e.g. Butler, 1998). A inclinação magnética pode também ser determinada a partir de tijolos, desde que sua forma geométrica e sua posição de queima em relação ao plano horizontal sejam conhecidas.

Para períodos de dezenas de ano até poucos milênios, o CMT é amplamente estudado a partir de medidas em materiais arqueológicos. O Arqueomagnetismo é uma área de pesquisa interdisciplinar que envolve Arqueologia, Magnetismo de Materiais e Geomagnetismo (Gallet et al., 2009). Uma aplicação do seu estudo consiste na utilização da "curva de referência" (que pode ser obtida diretamente dos dados ou de modelos de campo por harmônicos esféricos) para uma determinada região do globo como método de datação arqueológica. A chamada datação arqueomagnética consiste na comparação de um dado de um material magnético de idade desconhecida com uma curva de referência (Le Goff et al., 2002).

Neste trabalho foram estudadas as variações de inclinação magnética remanente em tijolos do sítio arqueológico Engenho Central de Piracicaba (ECP). Os dados foram comparados com o modelo do IGRF e aplicou-se a datação arqueomagnética em estruturas do Engenho em que não se conhece a que fase de ampliação elas correspondem, contribuindo assim com informações para a arquitetura do Engenho.

\section{Sítio arqueológico e metodologia}

O ECP foi construído à margem do Rio Piracicaba (Lat.: $22^{\circ} 43^{\prime} 8,4^{\prime \prime}$ Lon.: $\left.-47^{\circ} 39^{\prime} 22.7^{\prime \prime}\right)$ e durante o seu período de funcionamento (1881-1975 AD) passou por cinco fases de ampliação, sendo apenas as duas primeiras de idade conhecida. A figura 1 mostra a planta arquitetônica indicando as fases de construção de cada edificação do ECP. Para o estudo da variação da inclinação magnética dos tijolos, foram coletados fragmentos de tijolos e cilindros das edificações do ECP. A tabela 1 lista a localização dos pontos (paredes) amostrados in situ, as respectivas fases de ampliação e idades estimadas de acordo com Dutra \& Associados (2011) e o número de fragmentos coletados (tijolos inteiros e cilindros de tijolos).

As medidas de inclinação magnética foram determinadas através de técnicas de investigação da estabilidade dos portadores magnéticos e de identificação das componentes de magnetização. Para isso foi utilizada a desmagnetização por passos em campos magnéticos alternados (AF) e térmica de espécimes orientados dos tijolos. A identificação das componentes de magnetização será feita através de análises vetoriais e análise por componentes principais (Butler, 1998). As medidas de magnetização foram efetuadas no magnetômetro Superconducting Quantum Interference Device (SQUID) 


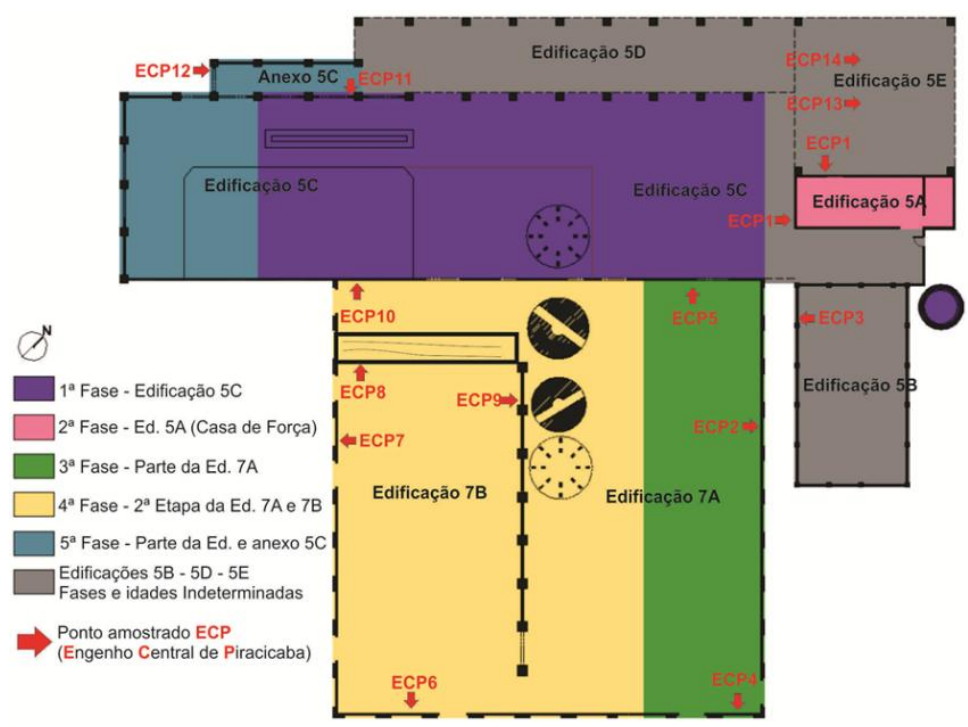

Figura 1 - Planta arquitetônica do ECP. A escala de cores mostra as 5 fases de construção e as setas em vermelho indicam os pontos onde foram amostrados os tijolos (modificado de Maria Luiza Dutra \& Associados, 2011).

U-channel da $2 \mathrm{G}$ Enterprises. A componente principal de magnetização é influenciada pela anisotropia magnética devido a técnicas de manufatura do tijolo. Para corrigir os possíveis efeitos de anisotropia magnética, foram feitas medidas do tensor de anisotropia de susceptibilidade magnética (ASM). Para determinação da estabilidade térmica e dos portadores de magnetização foram obtidas curvas de susceptibilidade magnética em baixos campos em ciclos de aquecimento e resfriamento em temperaturas que variam de $\sim 20^{\circ} \mathrm{C}$ a $700{ }^{\circ} \mathrm{C}$. Ambas as medidas de ASM e de curvas termomagnéticas foram efetuadas no susceptômetro Kappabridge KLY4 da AGICO.

Tabela 1 - Fases de ampliação e quantidade de amostras coletadas nos pontos amostrados do ECP. Os asteriscos $\left.{ }^{*}\right)$ indicam fases e idades desconhecidas.

\begin{tabular}{cccccc} 
& $\begin{array}{c}\text { Edificação } \\
\text { Ponto }\end{array}$ & do & Fase & Idade $(A D)$ & \multicolumn{2}{c}{ Qtde. de amostras } \\
ECP11 & 5C & $1^{\mathrm{a}}$ & 1881 & 6 & - \\
ECP5 & 5C & $1^{\mathrm{a}}$ & 1881 & - & 12 \\
ECP1 & 5A & $2^{\mathrm{a}}$ & $1910-1939$ & 15 & - \\
ECP2 & $7 \mathrm{~A}$ & $3^{\mathrm{a}}$ & $*$ & 12 & - \\
ECP4 & 7A & $3^{\mathrm{a}}$ & $*$ & - & 14 \\
ECP6 & 7B & $4^{\mathrm{a}}$ & $*$ & - & 10 \\
ECP7 & 7B & $4^{\mathrm{a}}$ & $*$ & - & 10 \\
ECP8 & 7B & $4^{\mathrm{a}}$ & $*$ & 8 & - \\
ECP9 & 7B & $4^{\mathrm{a}}$ & $*$ & 6 & - \\
ECP10 & 7B & $4^{\mathrm{a}}$ & $*$ & 5 & - \\
ECP12 & Anexo 5C & $5^{\mathrm{a}}$ & $*$ & 5 & - \\
ECP3 & 5B & $*$ & $*$ & 8 & - \\
ECP13 & 5E & $*$ & $*$ & 15 & - \\
ECP14 & 5E & $*$ & $*$ & 14 & - \\
\hline Total & & & & 94 & 46
\end{tabular}

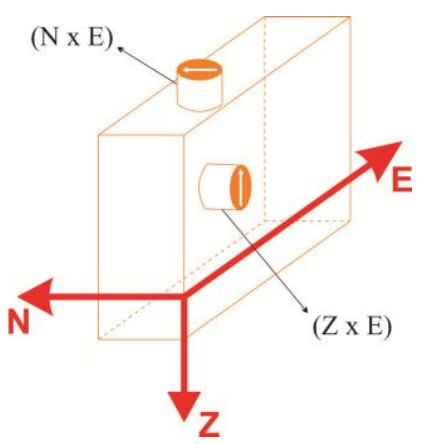

Figura 2 - Representação da preparação de espécimes em relação às faces planas do tijolo.

Os tijolos selecionados para medidas da inclinação possuem a forma geométrica de um paralelepípedo reto. Para determinar a inclinação magnética registrada em um tijolo assume-se que um de seus planos ( $\mathrm{N} \times \mathrm{E}$ ) (figura 2) é sempre queimado paralelo a horizontal. Para medidas de ASM e componentes principais, foram preparados espécimes perpendiculares aos planos $(Z \times E)$ e $(N \times E)$.

\section{Resultados}

A susceptibilidade magnética em ciclos de aquecimento e resfriamento foi efetuada em 27 amostras de tijolos do ECP. A figura 3 mostra dois exemplos de curvas de susceptibilidade magnética em ciclos de aquecimento e resfriamento até $700^{\circ} \mathrm{C}$. A figura 2a mostra um exemplo de uma amostra com valores de susceptibilidade magnética intermediário e reversibilidade no ciclo até 700 ${ }^{\circ}$ C. A figura $2 b$ apresenta uma curva com valores de susceptibilidade magnética mais alta. O comportamento magnético dos tijolos do ECP indica que a magnetização é estável em altas temperaturas, observado pela reversibilidade das curvas. 

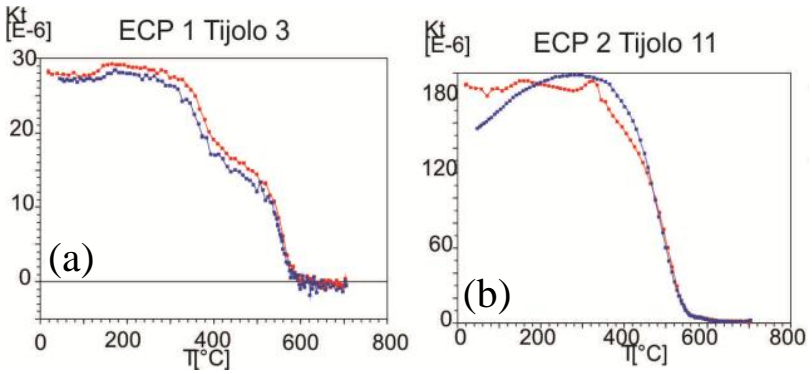

Figura 3 - Susceptibilidade magnética em ciclos de aquecimento e resfriamento até $700^{\circ} \mathrm{C}$.

As medidas de ASM foram feitas em 38 tijolos (201 espécimes) do ECP e os ângulos dos eixos principais de magnetização foram plotados em estereográficos, como mostra as figuras 4 e 5 . Foram medidos 168 espécimes preparados a partir do plano ( $\mathrm{E} \times \mathrm{Z}$ ) (figura $4 \mathrm{a}$ ) e as médias dos eixos com seus respectivos círculos de confiança de $95 \%$ são mostrados na figura 4b. Foram medidos 33 espécimes preparados a partir do plano (NxE) (figura 5a) e as médias dos eixos com seus respectivos círculos de confiança de $95 \%$ foram plotados na figura $5 b$.

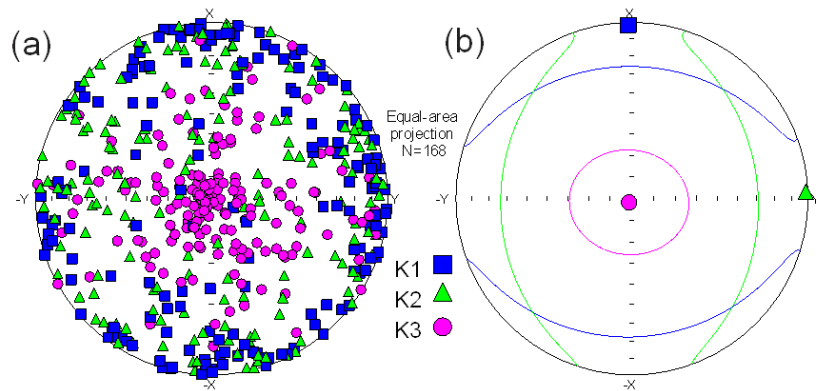

Figura 4 - Medidas de ASM para espécimes preparados no plano ( $E \times Z)$ do tijolo. (a) Valores dos ângulos de $K 1$, K2 e K3 das medidas de cada espécime. (b) Médias e círculos de confiança de 95\% para todos os espécimes.

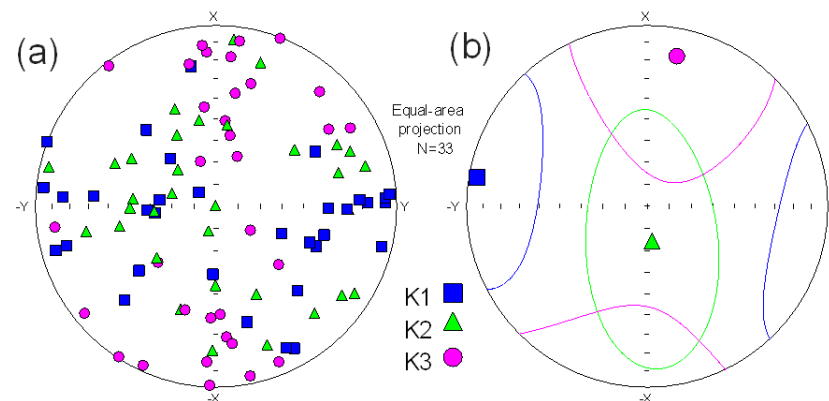

Figura 5 - Medidas de ASM para espécimes preparados no plano ( $N \times E)$ do tijolo. (a) Valores dos ângulos de $K 1$, K2 e K3 das medidas de cada espécime. (b) Médias e círculos de confiança de 95\% para todos os espécimes.

A partir dos gráficos pode-se definir que o eixo de menor susceptibilidade magnética (K3) é perpendicular ao plano $(E \times Z)$ e a direção dos eixos K1 e o K2 são aleatórios e perpendiculares ao eixo $\mathrm{K} 3$, devido ao processo de fabricação que prensa os tijolos sobre os planos ( $\mathrm{N} \times \mathrm{Z}$ ). O grau de anisotropia (K1/K3) varia entre $\sim 1.0$ e $\sim 1.08$.

As componentes de magnetização de 145 espécimes oriundas de 30 tijolos do ECP foram obtidas pela desmagnetização AF em 23 passos até 140 mT. Nenhum espécime desmagnetizou por completo até o campo máximo de $140 \mathrm{mT}$. A desmagnetização dos espécimes ECP02-11-1(NxE) e ECP01-03-3(NxE) apresentados, respectivamente, nas figuras $6 \mathrm{a}$ e $6 \mathrm{~b}$, são representativos dos outros espécimes dos tijolos do ECP em desmagnetizações AF.

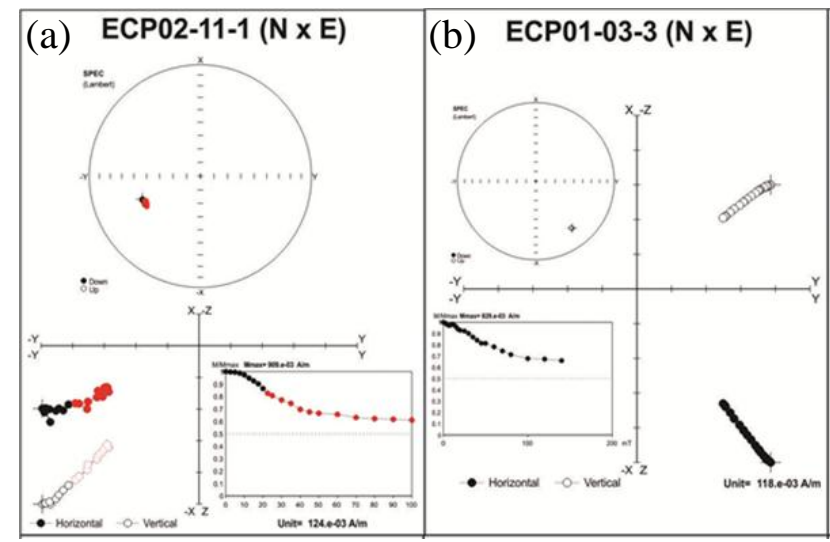

Figura 6 - Gráficos da desmagnetização por campos magnéticos alternados (AF) dos espécimes (a) ECP0211-1(NxE); e (b) ECP01-03-3(NxE).

As componentes de magnetização de 30 espécimes oriundas de 13 tijolos do ECP foram obtidas pela desmagnetização térmica em 20 passos de aquecimento até $650{ }^{\circ} \mathrm{C}$, sendo que todos os espécimes desmagnetizaram totalmente. A desmagnetização dos espécimes ECP08-01-2(NxE) e ECP03-02-4(NxE), apresentados, respectivamente, nas figuras $7 \mathrm{a}$ e $7 \mathrm{~b}$, são representativos dos outros espécimes dos tijolos do ECP que foram desmagnetizados termicamente.

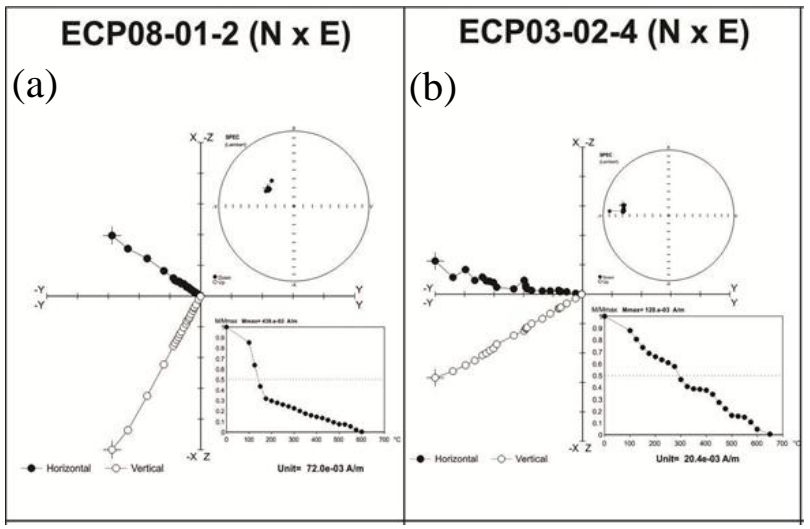

Figura 7 - Desmagnetização térmica em passos até 650 ${ }^{\circ} \mathrm{C}$ dos espécimes (a) ECP08-01-2(NxE); (b) ECP03-02$4(N x E)$.

Os espécimes desmagnetizados apresentaram magnetização da ordem de $\sim 10^{2}$ a $10^{\circ} \mathrm{A} / \mathrm{m}$ e componente 
magnética primária univetorial, como se pode verificar nos estereográficos das figuras 6 e 7. A magnetização dos espécimes é portada por grãos magnéticos de alta coercividade porque boa parte dos espécimes não desmagnetiza em campos magnéticos alternados até 140 $\mathrm{mT}$ (ver figura 6). O tratamento térmico desmagnetizou completamente os espécimes, comprovando 0 comportamento univetorial da magnetização dos tijolos.

Essas informações e as conclusões sobre a mineralogia magnética indicam que a magnetização é estável, sendo possível determinar as direções magnéticas de maneira confiável a partir das medidas de MRN. Foram determinadas as componentes principais a partir da MRN para de 125 espécimes oriundos de 38 tijolos. Tanto os valores de inclinação magnética obtidos pelas desmagnetizações AF e térmica, quanto os valores obtidos pela MRN foram utilizados para realizar as médias nesse sítio.

\section{Discussão e Conclusões}

A partir das medidas das componentes magnéticas determinadas pelas desmagnetizações AF e térmica e pelas medidas de MRN, foi possível determinar uma média dos dados de inclinação magnética para cada ponto amostrado no ECP. Para o cálculo da média foram utilizados os 38 tijolos (297 espécimes). A tabela 2 mostra os resultados de inclinação e os respectivos erros (a95) determinados a partir dos tijolos estudados. Após obter as médias, os valores foram comparados com o modelo do IGRF para a região para aplicação da datação arqueomagnética nos locais de idade desconhecida (figura 8).
Os resultados da datação arqueomagnética são apresentados na figura 9, onde se compara as idades com pontos de amostragem. O ponto ECP5 apresentou resultados de inclinação magnética com alto valor de a95 que influenciou na incerteza da datação arqueomagnética. Apesar do erro elevado esse ponto é definido, pelos estudos arquitetônicos e arqueológicos, como um dos mais antigos do ECP, assim como o ponto ECP11, oriundos da data de fundação do Engenho (1881 AD). Contudo, o valor obtido pela datação do ponto ECP11 não é coerente com essa informação de sua construção. Uma possível causa para essa diferença pode ser atribuída ao fato de restauração de tijolos posterior à construção inicial. O ponto ECP1 (Casa de Força) possui uma datação coerente com as informações arquitetônicas, sendo que ele foi construído entre 1921 AD e 1936.5 AD. Para o ponto ECP2 foi obtido o intervalo de 1926.5-1935 AD, indicando que sua construção foi contemporânea a construção da Casa de Força (ECP2). Os dados do ponto ECP8 não apresentam confiabilidade necessária para definição de um intervalo de idade. Isso ocorre porque o ECP8 apresenta apenas um tijolo medido de maneira que os desvios na inclinação não são eliminados. O ponto ECP9 possui a maior incerteza entre os pontos medidos devido aos valores elevados do a95. Contudo, pode-se inferir que os pontos ECP 11, ECP 1, e ECP2 são contemporâneos a uma mesma fase de ampliação. A idade e a fase de construção do ponto ECP3 não são conhecidas pelos estudos arquitetônicos do ECP. Através da datação arqueomagnética é possível afirmar que a idade desse ponto esteja entre $1931.5 \mathrm{e}$ 1939.5 AD, sendo contemporâneo aos pontos ECP9 e ECP10.

Tabela 2 - Informações dos pontos de amostragem do ECP. Na tabela são apresentados o número de cilindros, tijolos e espécimes utilizados para obtenção das médias de inclinação magnética e os respectivos a95. São apresentados também os intervalos de idade calculados a partir dos dados medidos e os calculados pelo IGRF.

\begin{tabular}{|c|ccc|cc|cc|} 
& \multicolumn{3}{|c|}{$N^{\circ}$ utilizado de } & \multicolumn{3}{c|}{ Inclinação } & \multicolumn{2}{c|}{ Datação (IGRF) } \\
Ponto & Cilindros & Tijolos & Espécimes & Média & $\alpha 95$ & Início & Fim \\
\hline ECP 5 & 6 & - & 16 & -13.38 & 6.35 & 1881 & 1937.5 \\
ECP 8 & - & 1 & 8 & -17.13 & 1.55 & 1936.5 & 1945.5 \\
ECP 1 & - & 8 & 74 & -15.43 & 2.16 & 1921.5 & 1936.5 \\
ECP 11 & - & 6 & 39 & -15.34 & 1.42 & 1924.5 & 1933.5 \\
ECP 2 & - & 10 & 84 & -15.68 & 1.41 & 1926.5 & 1935.5 \\
ECP 10 & - & 5 & 35 & -16.58 & 1.84 & 1931.5 & 1942.5 \\
ECP 3 & - & 4 & 21 & -17.01 & 3.28 & 1931.5 & 1949.5 \\
ECP 9 & - & 4 & 20 & -19.54 & 7.82 & 1931.5 & 1972.5 \\
\hline Total & 6 & 38 & 297 & \multicolumn{3}{|c|}{}
\end{tabular}




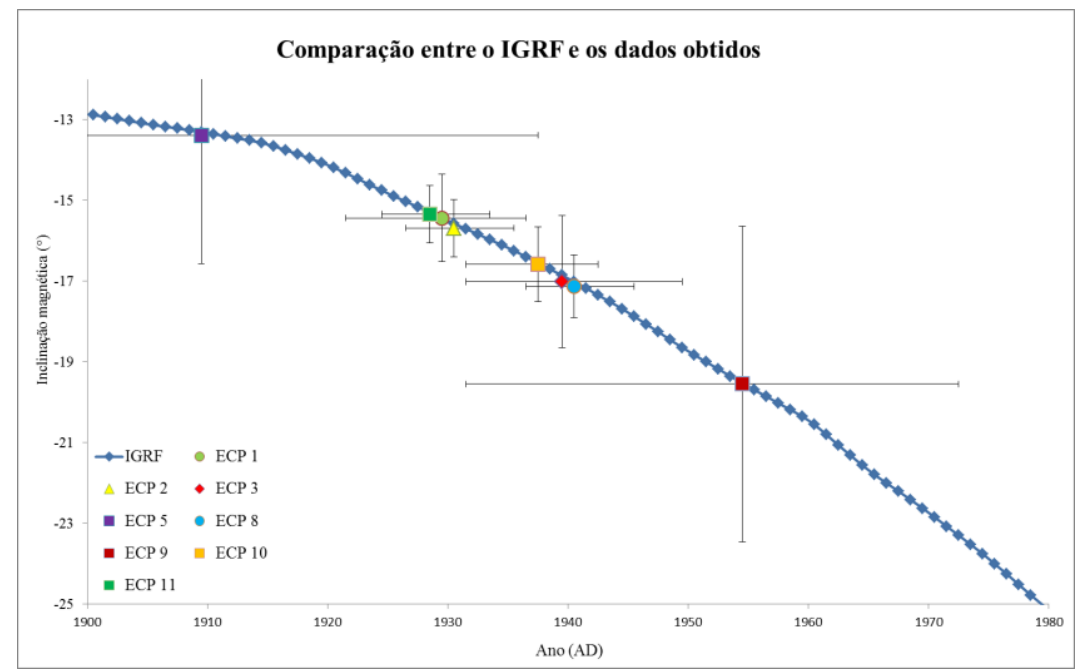

Figura 8 - Gráfico de datação arqueomagnética que compara os valores obtidos das medias de inclinação magnética (tabela 2) com a curva do modelo do IGRF de inclinação magnética (curva azul).

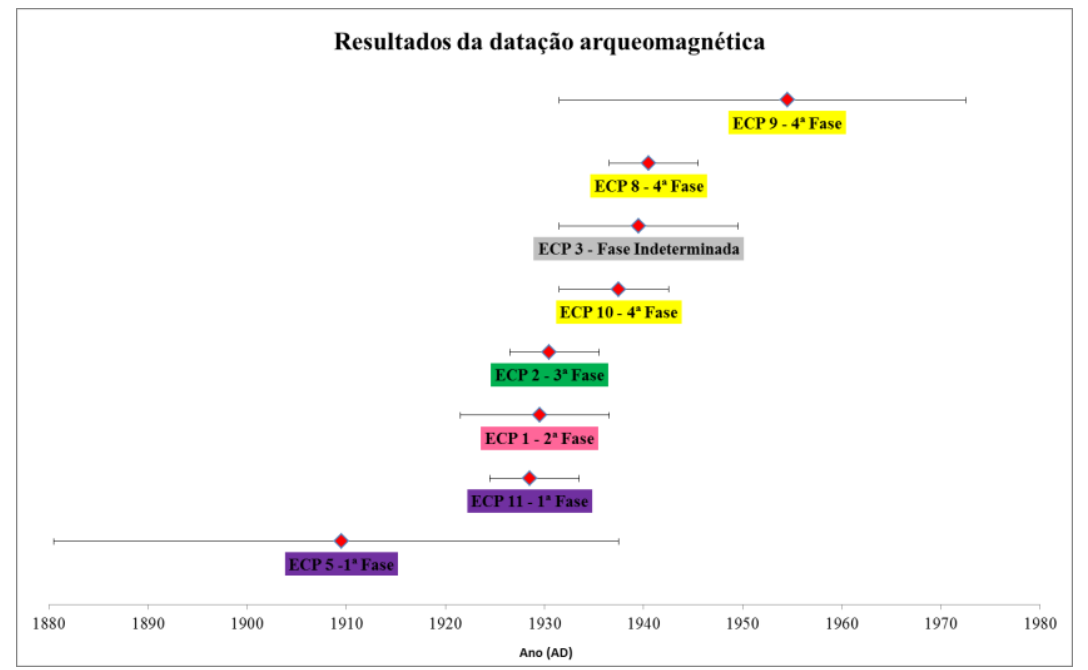

Figura 9 - Resultados e incertezas obtidas através da datação arqueomagnética a partir do modelo do IGRF.

\section{Agradecimentos}

Agradeço a Sociedade Brasileira de Geofísica (SBGf) por financiar este trabalho através de bolsa de Iniciação Científica para graduação.

\section{Referências}

Butler, R.F., 1998. Paleomagnetism: Magnetic Domains to Geological Terranes. Blackwell Scientific Publications.

Dutra, M.L. \& Associados, 2011. Museu do Açúcar e do Álcool, Projeto Executivo de Restauro, Relatório para pesquisa arqueológica.
Gallet, Y. et al (03 co-authors), 2009. Geomagnetic field hemisphere asymmetry and archeomagnetic jerks. Earth Planet. Sci. Lett., 284: 179-186.

Le Goff, M. et al (03 co-authors), 2002. On archeomagnetic secular variation curves and archeomagnetic dating. Phys. Earth Planet. Int., 134: 203211. 